\title{
A case of seminoma presented with clinical manifestations of testicular torsion
}

\author{
Aytac Sahin ${ }^{1}$, Caglar Yildirim ${ }^{1}$, Serkan Akan ${ }^{1}$, Ozgur Haki Yuksel ${ }^{1}$, Ahmet Urkmez ${ }^{2}$ \\ ${ }^{1}$ Fatih Sultan Mehmet Research \& Training Hospital, Dept. of Urology, Istanbul, Turkey; \\ ${ }^{2}$ Haydarpasa Numune Research \& Training Hospital, Dept. of Urology, Istanbul, Turkey.
}

\begin{abstract}
Summary Testicular tumors rarely manifest themselves with clinical picture of testicular torsion.

In this presentation of ours, we reported a 30-year-old patient whose post-orchiectomy histopathology report revealed the presence of seminoma. The patient consulted us with acute scrotum whose physical examination and Doppler ultrasonographic findings showed testicular torsion. Though rarely seen patients, in cases who consulted with acute scrotum, the possibility of testicular tumor should not be discarded. For the establishment of differential diagnosis detailed anamnesis and physical examination findings should be supported with laboratory tests and imaging modalities.
\end{abstract}

KEY WORDS: Testicular torsion; Seminoma; Orchiectomy.

Submitted 2 February 2017; Accepted 5 May 2017

\section{INTRODUCTION}

Testicular torsion is a scrotal emergency case occurs as a result of rotation of spermatic cord around its axis leading to impairment of testicular perfusion and testicular ischemia. Scrotal pain spreading into the lower abdominal quadrant can cause concomitant symptoms of nausea and vomiting. The incidence rate of testicular torsion is around 3.5/100000. It is mostly idiopathic and 20\% of the cases are related to trauma (1). Torsion is a scrotal phenomenon, which can be observed in adolescents.

$1-2 \%$ of all malignant tumors seen in men are testicular cancers and incidence rate of testicular cancers increases among young men aged between $15-40$ years old. $80 \%$ of testicular tumors are seminomas, and most of them present themselves as clinical stage I disease. These two scrotal diseases can complicate each other, torsion due to testicular tumor may occur, however the possibility of simultaneous occurrence of these two diseases in the same testis is very low. Scrotal color-Doppler ultrasound (CDUS) has a major role for differential diagnosis, however despite higher rates of diagnosis, in case of uncertainty, taking a decision to perform surgical exploration and orchiectomy without delay is essential. In this presentation of ours, we reported a 30-years-old patient whose post-orchiectomy histopathology report shows the presence of seminoma. The patient consulted to us due to acute scrotum. His physical examination and Doppler ultrasonographic findings were consistent with testicular torsion.

\section{Case report}

A 30 years-old male patient, has two children, applied to the emergency service due to left testicular pain lasted for a day. The patient stated that his pain started suddenly without any history of trauma. Also there was not any similar attack before. Besides the patient doesn't have any known systemic comorbidity. On physical examination left testis seen oedematous and scrotal skin overlying the left testis was hyperaemic. Left hemiscrotum was extremely tender on palpation and comparing with its counterpart, the left testis was seem to be elevated because of the inflammation. His urinalysis was unremarkable. Leukocytosis (16.000/ml, normal range 4600$10200 / \mathrm{ml}$ ), extremely high levels of serum lactic acid dehydrogenase (LDH) (650 U/L, normal range 125220U/L), and C-reactive protein $(9 \mathrm{mg} / \mathrm{dl}$, normal range $0-0,5 \mathrm{mg} / \mathrm{dl}$ ) were detected. Serum beta- HCG, and alfafetoprotein (AFP) values were within normal limits.

Left testis demonstrated a heterogeneous structure on scrotal CDUS obtained in the emergency service and blood flow was not detected. CDUS images suggested testicular torsion. The spermatic cord manually detorsed prior to his surgical procedure but his clinical signs didn't change. We talked about possible orchiectomy with the patient and his family. There was no suspicion of testicular tumor prior to orchiectomy. Our main surgical intervention purpose was treatment of testicular torsion, so we performed scrotal exploration under general anesthesia immediately. Testicular blood flow was not observed and no/any rotation wasn't observed at cord. Testis and spermatic cord were wrapped with warm compress for 15 minutes. Then testicular parenchyma was controlled and any evidence of blood flowing was not detected. Tunica vaginalis was incised with a surgical blade to detect if there is any bleeding. Testicular bleeding on the exploration is important, because it shows that there is still circulation to the testis. But, just purulent and necrotic fluid was drained (Figure 1). For microbiological analysis specimens were taken and then scrotal orchiectomy was performed. The patient was discharged on postoperative 1. day, there was not any complication depending on the surgery. Histopathology results were reported as granulomatous inflammation and seminoma (Figure 2). CT obtained at postoperative 2 weeks, metastatic lesion in the lungs was not detected. Serum LDH levels regressed to $121 \mathrm{U} / \mathrm{L}$. Adjuvant chemotherapy was planned 
for the patient with the diagnosis of pT1NOMO seminoma and medical oncological treatment was initiated. Now the patient is still monitored by the oncologist.

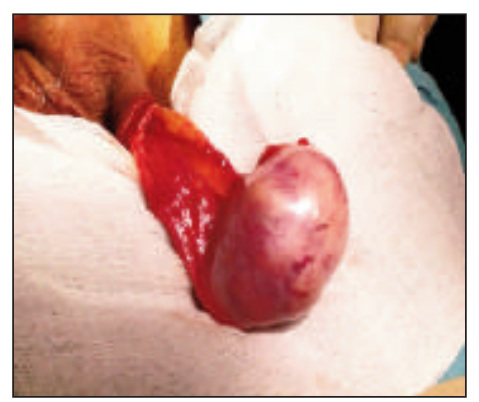

Figure 1.

During surgical exploration nontorsioned cord, heterogenous testicular structure, and necrotic, and purulent discharge observed when vaginal tunica was incised with a scalpel.

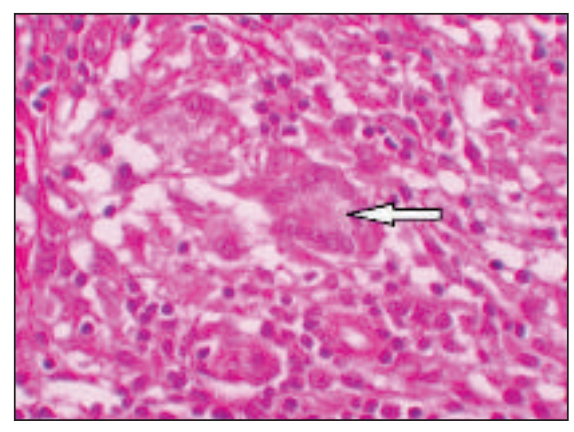

Figure 2.

Microscopic examination reveals patchy areas of diffuse groups of tumoral cells associated with small lymphocytes in a fibrous stroma. Tumoral cells consist of atypical germ cells with clear or eosinophilic cytoplasm, generally uniform large round nuclei with thin chromatin, marked nucleoli, and mitotic figures. Granulomatous reaction partly associated with tumor is seen.

\section{Discussion}

Testicular torsion is a scrotal emergency disease, which occurs before the age of 20 and characterized by sudden onset of testicular pain. The ratio of testicular torsion among men aged over 20 years in the literature reported cases varies between percent 10 and 56 of all cases (2). Our case was 30 years old who can be considered as over aged by comparing the average age mentioned in the literature of testicular torsion. Acute testicular pain is considered as testicular torsion unless proved otherwise. Testicular parenchyma damage starts to take effect within the first 4 hours of occlusion of testicular veins/arteries after the torsion of the spermatic cord. Therefore in case of testicular torsion suspect, one should not hesitate to perform testicular exploration. On physical examination even some findings suggest testicular torsion, concomitant testicular swelling or hydrocele may mask testicular torsion. In testicular torsion the involved testis is solid, tender and tense and it can be elevated because of the shortening of the spermatic cord of the affected testis when compared with its counterpart. If testicular pain is relieved with testicular elevation then probability of epididymo-orchitis is present. In spermatic cord torsion cases, testicular pain is not relieved after manual elevation of the testis. Cremaster reflex is not seen in testicular torsion, however cremaster reflex can be seen in the torsion of appendix testis, and it can show symptoms similar to those seen in cases with torsion of the spermatic cord. In our case the involved testis was tender to touch and elevated relative to other testis and there was no cremaster reflex. Urinalysis is generally unremarkable and 50\% leukocytosis can be seen. In our case leukocytosis was present, while results of urinalysis were within normal limits.

Since most of the scrotal diseases show similar symptoms, clinical diagnosis of testicular torsion is a challenging issue. CDUS is a reliable method in the accurate diagnosis of scrotal pathologies. In addition to scrotal pathologies, CDUS can also comparatively evaluate normal scrotal anatomy. Becker et al. reported higher diagnostic sensitivity (90.5\%), and specificity (98.3\%) of scrotal CDUS for testicular torsion (3). Despite all physical examination findings and test results, if clinical suspicion still persists, then surgical exploration should not be declined. As is the case with other organ tumors, organ-preserving surgeries are revived for testicular tumors, however in cases with normal contralateral testis, this approach is not recommended. Besides, biopsy is not a suitable option because of causing a possible shift in the lymphatic pathway and a risk of false positivity testicular. In patients for whom orchiectomy is decided, testicular tumor should be suspected, and spermatic cord should be clamped at the level of internal ring of the inguinal canal, and high-level orchiectomy should be performed. Indeed CDUS images of our patient did not demonstrate apparent characteristics of a testicular tumor, and any evidence of testicular blood flow was not detected. So we performed scrotal exploration to treatment of torsion. The patient underwent orchiectomy with the indication of torsion, and histopathology report of the specimen indicated pTl seminoma.

\section{Conclusion}

For the establishment of differential diagnosis detailed anamnesis, and physical examination findings should be reinforced with laboratory tests, and imaging modalities. Even if all preoperative examinations and tests indicate testicular torsion, clinical common sense should never rule out the possibility of testicular cancer.

\section{References}

1. Huang WY, Chen YF, Chang HC, et al. The incidence rate and characteristics in patients with testicular torsion: a nationwide, population-based study: Acta Paediatr. 2013; 8:363-367.

\section{Althaffer LF. Testicular torsion in men. J Urol. 1980; 123:37.}

3. Becker D, Burst M, Wehler M, et al. Differential diagnosis of acute testicular pain using color-coded duplex ultrasonography: difference between testicular torsion and epididymitis. Dtsch Med Wochenschr. 1997; 122:1405-1409.

\section{Correspondence}

Aytac Sahin, MD - Caglar Yildirim, MD

Serkan Akan, MD - Ozgur Haki Yuksel, MD

Fatih Sultan Mehmet Research\& Training Hospital,Dept. of Urology Istanbul, Turkey

Ahmet Urkmez, MD (Corresponding Author)

ahmeturkmez@hotmail.com

Haydarpasa Numune Research\& Training Hospital,Dept. of Urology Uskudar Tr- 34668 Istanbul, Turkey 